\title{
Miquel Siguan*
}

\author{
Universidad de Barcelona
}

\section{LOS ANTECEDENTES}

Precisamente porque al tratar este tema acostumbran a encresparse los ánimos, son tan necesarios los datos objetivos sobre lo que está ocurriendo y la investigación con rigor científico de los resultados producidos. Afortunadamente, tales datos y tales investigaciones empiezan ya a estar disponibles y aptas para ser comentadas, y esto es lo que me propongo hacer. Pero antes de iniciar el comentario parece conveniente intentar resumir brevemente la situación en la que se encuentra el problema.

El catalán, que en la Edad Media era floreciente medio de expresión de una comunidad política importante y que animó una floreciente literatura, logró resistir unas circunstancias históricas adversas a lo largo de varios siglos y mantenerse como lengua de uso común hasta conocer, en el siglo pasado, un renacimiento esplendoroso unido a un desarrollo económico de Cataluña y. a una renovada conciencia política. En la actualidad Cataluña dispone de una amplia autonomía polírica y de un gobierno propio. En cuanto a la lengua, no sólo el catalán tiene el carácter de lengua cooficial junto con la castellana en el ámbito de Cataluña, sino que el gobierno y las instituciones públicas catalanas están decididamente dispuestas a apoyar la lengua catalana y la cultura que con ella se expresa.

A pesar de todo ello, la pervivencia del catalán sigue siendo problemática.

El primer hecho a tener en cuenta es naturalmente el mayor peso de la lengua cas- tellana, que no sólo es lengua oficial del Estado español sino el medio de comunicación y de expresión de una comunidad humana mucho mayor que la de los catalano-hablantes. Así, aunque Barcelona sea el centro de la producción de libros en catalán y la producción de libros en catalán sea relativamente abundante en Barcelona, se editan muchos más libros en lengua castellana. $Y$ aunque se publiquen periódicos, revistas y se produzcan películas en catalán, el público de Cataluña adquiere muchos más periódicos y revistas en castellano y contempla muchas más películas en castellano que en catalán. También el número de horas de TV emitidas en castellano es todavía mayor que el de emitidas en catalán, aunque exista una TV pública en catalán.

$\mathrm{Y}$ en segundo lugar hay que tener en cuenta un hecho demográfico. Si bien es cierto que el desarrollo económico de Cataluña ha potenciado su personalidad políti$\mathrm{ca}$, también lo es que ha provocado una inmigración masiva especialmente del sur de España -y por tanto de lengua castellana-, hasta el punto de que en la actualidad cerca de la mitad de la población de Cataluña tiene el castellano como lengua materna o familiar, proporción que a su vez está muy diversamente repartida: mientras en la ciudad de Barcelona se da un cierto equilibrio entre los dos orígenes lingüísticos, en las zonas rurales predomina claramente el catalán y en el cinturón industrial de Barcelona el predominio del castellano es completo.

En estas circunstancias puede hablarse de una siruación diglósica en favor del caste- 
llano, aunque se trate de una situación diglósica singular, pues algunos indicadores clásicos de la diglosia no se cumplen o lo hacen en forma paradójica. Así es cierto que, en la medida en que Cataluña forma parte del Estado español, el castellano es la lengua del poder político y por tanto tiene un prestigio superior, mientras el catalán es la lengua de una minoría lingüística, lo que es típico de una situación diglósica. El hecho de que la presencia del catalán sea mucho más fuerte en el campo que en la ciudad también se corresponde con una situación de este tipo. Pero al mismo tiempo el hecho de que el catalán sea mayoritario en las clases medias, incluyendo a los intelectuales y a una buena parte de la burguesía, mientras el castellano es más frecuente entre los trabajadores industriales -especialmente entre los menos calificados-y entre los sectores sociales claramente marginados, apuntaría a una diglosia de signo inverso con el catalán como lengua de más prestigio.

Hay en cambio otro indicador de diglosia, menos utilizado en los estudios sobre bilingüismo, pero que en este caso es muy revelador del desequilibrio en favor del castellano: me refiero a la proporción de bilingües entre los hablantes de las dos lenguas, proporción que efectivamente es muy diversa. Prácticamente todos los catalanes entienden y hablan el castellano, mientras sólo una parte de los castellano-hablantes pueden hacer lo mismo con el catalán (en líneas muy generales podrá decirse que de los habitantes de Cataluña que tienen el castellano como primera lengua un $25 \%$ entienden y hablan el catalán, un $50 \%$ lo entienden pero no lo hablan y un $25 \%$ ni lo hablan ni lo entienden). En estas condiciones una comunicación entre dos o varios interlocutores de los que alguno sea castellanohablante se establece, casi espontáneamente, en castellano.

En cuanto a la presencia del catalán en el sistema educativo la situación puede resumirse brevemente así:

La política lingüística en la educación del gobierno de la Generalitat -al que por el Estatuto de Autonomía se han transferido todas las competencias en materia de educación- se propone que al término de la Educación General Básica los escolares sean capaces de usar normalmente las dos lenguas tanto en la comunicación oral como en la escrita. Y dado que se ha partido de una situación de predominio del castellano - $\mathrm{O}$ mejor dicho, ausencia total del catalán en la enseñanza- su actuación se ha dirigido principalmente a potenciar el papel del catalán para hacer posible aquel objetivo. Elementos de esta actuación han sido la introducción del catalán en las Escuelas de Magisterio, el reciclaje en catalán de los docentes en ejercicio, el estímulo a las escuelas dispuestas a desarrollar la enseñanza en catalán, etc.

Más concretamente, la presencia del catalán y del castellano en la enseñanza está regulada por una serie de disposiciones de las cuales las primeras que establecen la obligatoriedad de la enseñanza del catalán en todos los grados y modalidades de la enseñanza datan de 1978 y son por tanto anteriores a la autonomía política de Cataluña y al Estatuto que la regula. El conjunto de disposiciones vigentes en la actualidad puede resumirse diciendo que se admite una amplia variedad de planteamientos lingüísticos desde la escuela con enseñanza predominantemente en castellano hasta la escuela con enseñanza predominantemente en catalán, pero que en cualquier caso a la lengua menos utilizada hay que dedicarle al menos de tres a cinco horas semanales, en todos los niveles, grados y modalidades de la enseñanza. La existencia de estos mínimos y el esfuerzo del Gobierno de Cataluña por aumentar el uso del catalán como lengua de enseñanza, constituyen así los rasgos más destacados para definir la situación actual.

A ellos puede añadirse la opinión generalizada de que la introducción en la enseñanza conviene hacerla en la lengua materna y familiar del niño. Tradicionalmente, por esta introducción se entendía la adquisición de la lectura y la escritura, pero en la actualidad la generalización de la educación preescolar ha variado el planteamiento del problema. El hecho de que en el aula de prescolar convivan niños de lengua familiar catalana y de lengua familiar castellana y que el enseñante se dirija a cada uno en su lengua produce una familiaridad temprana con la segunda lengua, de tal modo que incluso para los niños que se inician en la lec- 
tura en la segunda lengua la iniciación no plantea dificultades especiales.

Finalmente el último y muy importante dato a tener en cuenta es que, aunque actualmente en Cataluña existan distintos tipos de escuelas según el predominio de una u otra lengua en la enseñanza, esto no significa la existencia, como ocurre en otros lugares, de dos sistemas educativos distintos para dos poblaciones lingüísticamente distintas, sino que en cualquier escuela se pueden encontrar alumnos de una y otra lengua familiar.

¿En qué se han traducido en la práctica estas disposiciones y cuáles son los resultados conseguidos?

Hace algún tiempo apareció con el título Lenguas y Educación en el ámbito del Estado Español el volumen que recoge las ponencias presentadas al Seminario sobre este tema que anualmente y desde hace ocho años organiza en Sitges el Instituto de Ciencias de la Educación de la Universidad de Barcelona y que en este momento constituye el conjunto de información sobre este tema más completo y puesto al día disponible. Entre otros muchos datos de interés, se puede leer en una ponencia presentada por la propia dirección de Enseñanza de la Consellería de Educación del gobierno catalán que, en 1982, del total de los centros de EGB en Cataluña, un $78 \%$ ofrecían una enseñanza en castellano y un $21 \%$ una enseñanza total o parcialmente en catalán.

Pero en el propio volumen se incluye además el resumen de un estudio de valoración de los resultados conseguidos realizado por el gabinete de estudios del Servicio de Enseñanza del Catalán de la propia Dirección General, Cuatro años de catalán en la escuela: factores y resultados. Posteriormente se ha publicado también el estudio completo con el título Quatre anys de català a l'escola y en él se centra el presente comentario.

\section{METODOLOGIA}

Antes de abordar el comentario de los resultados conseguidos es necesario exponer con algún detalle la metodología utilizada en el estudio.
Objetivos: El estudio tenía por objeto valorar el nivel de dominio del catalán y del castellano alcanzado por los alumnos al llegar al cuarto año de la EGB y poner en relación este nivel con el grado de presencia de las dos lenguas en la escuela y con el conjunto de otras variables que podían influir en los niveles alcanzados por los alumos.

Características de la muestra: Teniendo en cuenta que, como ya he señalado, las escuelas difieren ampliamente según la proporción en que utilizan las dos lenguas en la enseñanza y que son muy diferentes también las proporciones de hablantes de una y otra lengua según las escuelas y según las poblaciones, se decidió construir la muestra teniendo en cuenta estas dos variables y distinguiendo en cada una de ellas dos niveles:

Grado de catalanización de la escuela: Enseñanza predominantemente en catalán, enseñanza predominantemente en castellano, situaciones intermedias.

Grado de catalanización de la población donde está ubicada la escuela: Poblaciones con predominio de catalano-parlantes, poblaciones de relativo equilibrio.

Combinando los dos criterios se establecieron nueve tipos de escuelas. A continuación se clasificaron todas las escuelas de $\mathrm{Ca}$ taluña según estos nueve tipos y se eligieron al azar seis escuelas en cada tipo, lo que produjo una muestra de 54 escuelas esparcidas por todo el territorio catalán. En estas escuelas se examinaron todos los alumos que estaban cursando el cuarto año de EGB prescindiendo de los que habían sido alumnos de la escuela menos de dos años y algunos otros que por distintas razones no pudieron efectuar las pruebas. Quedaron finalmente 1.539 alumnos que son los que constituyeron la muestra examinada.

Como ya ha quedado claro por lo que acabo de exponer, no se trata de una muestra representativa de la totalidad de la población escolar de Cataluña, sino representativa de los distintos tipos de escuelas para poder comparar entre sí los resultados conseguidos.

Características de los sujetos: Para estos 1.539 sujetos se obtuvieron una serie de datos susceptibles de influir en los resultados conseguidos y destinados a correlacionarlos con éstos. Estos datos fueron: Sexo, lugar de nacimiento (dentro o fuera de Cataluña), 

(alta, media, baja) lengua familiar (catalán, castellano o las dos), condición lingüística ambiental de la escuela (por condición lingüística ambiental se entendía el predominio de una u otra lengua entre los alumnos de la escuela considerada y se tomaba como medida la media de las características lingüísticas de los alumnos del mismo centro docente), actitud ante las lenguas (deducida de las respuestas del sujeto a un cuestionario) y evaluación por el propio sujeto de su competencia en cada una de las dos lenguas. Finalmente cada sujeto recibió una calificación denominada «capacidad de aprendizaje» y que era la media de sus calificaciones escolares en el curso anterior.

Pruebas lingüisticas: A cada uno de los sujetos se le aplicaron un conjunto de pruebas paralelas en catalán y castellano, destinadas a evaluar su competencia en cada una de las dos lenguas. Dado que la competencia lingüística tiene aspectos muy diversos, la elección del conjunto de pruebas que han de permitir su medida, así como el peso que se atribuye a cada una de ellas en la evaluación conjunta tiene siempre un margen de arbitrariedad. En el caso de este estudio, sus autores se esforzaron por cubrir un espectro amplio de la competencia lingüística y no limitarse a los aspectos más tradicionalmente escolares. El detalle de las distintas pruebas utilizadas puede verse en la publicación que he citado, aquí me limito a su enumeración.

- Comprensión oral: Palabras y frases aisladas.

- Compresión lectora: Palabras y frases aisladas.

- Expresión oral: Léxico, competencia comunicativa.

- Expresión escrita: Producción de un texto (composición).

- Fonología: Capacidad de emitir ciertos fonemas.

- Interferencias: Léxicas, semánticas y morfosintácticas.

- Ortografía.

- Fonética: Tipo de acento.

RESULTADOS LINGUISTICOS

DE LA ENSEÑANZA

Los resultados de la investigación pueden resumirse así:

Los niños de lengua materna y familiar castellana que asisten a una escuela en la que la enseñanza se da predominantemente en castellano, adquieren el nivel de competencia en esta lengua que se considera normal según los programas educativos. Pero los que asisten a una escuela en la que la enseñanza se da predominantemente en catalán, a pesar de que su exposición escolar al castellano es mucho menor, alcanzan prácticamente los mismos niveles.

Su nivel de adquisición del catalán en cambio es muy diverso según el tipo de escuela a la que asisten. Los que asisten a una escuela de enseñanza predominantemente en castellano, a pesar de que reciben regularmente la enseñanza del catalán prescrita por la reglamentación vigente, consiguen resultados bastante pobres o muy pobres, tanto en su capacidad de expresión oral como escrita en esta lengua. Sólo los que asisten a una escuela donde la enseñanza es mayoritariamente en catalán adquieren un dominio del catalán que pueda compararse con su dominio del castellano.

En cuanto a los niños de lengua materna y familiar catalana, si asisten a una escuela en lengua castellana, al cabo de un tiempo de escolaridad alcanzan un conocimiento del castellano que, medido por medio de pruebas de lenguaje, es comparable al que alcanzan sus compañeros en lengua castellana. Si asisten a una escuela catalana, a pesar de que sólo reciben el mínimo reglamentario de enseñanza de y en castellano, consiguen un nivel de competencia en esta lengua parecido o sólo ligeramente inferior.

Su nivel de competencia en catalán depende en cambio del tipo de escuela a la que asisten. Si asisten a una escuela en lengua castellana mantienen su dominio de la competencia oral y adquieren una buena comprensión de la lengua escrita, pero su vocabulario catalán crece menos que el castellano y sobre todo su capacidad de expresión escrita en catalán es inferior a la que adquieren en castellano. Sólo los niños de lengua materna catalana que asisten a una escuela con enseñanza en parte o predomi- 
nantemente en catalán adquieren una competencia escrita comparable en las dos lenguas.

Es decir que el sistema educativo en Cataluña desde un punto de vista lingüístico es simétrico pero sus resultados no son simétricos: en las escuelas predominantemente en catalán los alumnos adquieren unas competencias lingüísticas en castellano muy superiores a las competencias lingüísticas en catalán que adquieren los alumnos de las escuelas predominantemente en castellano.

Esta disimetría tiene naturalmente razones externas a la escuela. Los niños de lengua materna y familiar catalana llegan a la escuela con un cierto bagaje de castellano que han recibido de su ambiente, y todo a lo largo de su escolaridad su medio ambiente social les seguirá impulsando a un uso más o menos frecuente del castellano en múltiples circunstancias. En cambio el niño de lengua materna y familiar castellana, en la mayoría de los casos llega a la escuela ignorando totalmente el catalán y a lo largo de su escolaridad las presiones de su ambiente para que lo utilice serán pocas.

Los resultados del estudio que comento permiten formular esta disimetría y el desequilibrio que de ella resulta en otra forma más original y no menos ilustrativa.

Los niños de lengua materna catalana no sólo, como he dicho, consiguen unos resultados en lengua castellana que en su conjunto son comparables con los que consiguen los niños de lengua castellana, sino que, para cada niño, las calificaciones que consigue en cada una de las dos lenguas son relativamente parecidas y las dos a su vez se correlacionan con su calificación académica general. Dicho en forma simplificada, esto quiere decir que entre los niños de lengua materna catalana los mejores estudiantes obtienen buenas calificaciones tanto en castellano como en catalán y, a la inversa, los peores estudiantes tienen malas calificaciones en las pruebas de lenguaje tanto en catalán como en castellano. Este hecho es más claro en los alumnos de las escuelas de lengua catalana, pero en cierta medida se da también en las predominantemente en lengua castellana. $O$ sea que no se produce el llamado efecto balancín, según el cual la ganancia en competencia en una len- gua se hace a costa de la otra. En cambio, en los alumnos de la lengua materna castellana este hecho no se produce o en todo caso sólo entre los que asisten a escuelas mayoritariamente en catalán, para los demás sus calificaciones en catalán son prácticamente independientes de sus calificaciones en castellano.

La razón de este hecho, a primera vista sorprendente, no parece difícil de dar. El niño de lengua materna catalana por influencia de su ambiente se convierte prácticamente en bilingüe y dispone de dos instrumentos lingüísticos que maneja prácticamente sin dificultad. A partir de aquí sus progresos en las dos lenguas y especialmente en los aspectos de la competencia que miden las pruebas: vocabulario, sintaxis, ortografía... pueden ser relativamente paralelos y relacionados con sus aptitudes intelectuales y con sus actitudes escolares. En cambio el niño de lengua materna castellana con un conocimiento limitado del catalán y con una práctica reducida, el nivel de competencia que adquiere en el catalán más que de sus aptitudes intelectuales o de sus comportamientos escolares depende de la frecuencia con que entra en contacto con esta lengua en su vida cotidiana y de sus actitudes ante ella.

\section{CONSECUENCIAS PARA UNA POLITICA LINGUISTICA EN LA EDUCACION}

De los resultados expuestos se deduce que mientras la escuela predominantemente en lengua catalana, alcanza el objetivo de que sus alumnos al término de la enseñanza posean una alta competencia en una y otra lengua, la escuela predominantemente en lengua castellana, a pesar de ofrecer una enseñanza continuada del catalán, no permite alcanzar este objetivo y que concretamente el nivel de competencia en catalán que alcanzan sus alumnos de lengua materna castellana es claramente insuficiente. Sólo si el catalán además de lengua enseñada es, en alguna medida, lengua en la que se enseña, se produce el estímulo para usarla en la comunicación oral y escrita y así aumentar su competencia.

La consecuencia para la política educati- 
va parece clara: si se quiere alcanzar el objetivo propuesto de dominio de las dos lenguas al final de la E.G.B. es necesario aumentar la presencia del catalán en las escuelas de enseñanza predominantemente en castellano. Este es el fundamento de la disposición reciente a la que hemos aludido.

Si bien es cierto, que esta consecuencia puede matizarse desde distintas argumentaciones.

Es posible pensar que los resultados del estudio que he expuesto y, concretamente, los buenos resultados de los alumnos de lengua castellana en la escuela catalana, pueden explicarse por ciertas características de la población estudiada que no son forzosamente generalizables. Es un hecho que en las escuelas catalanas donde se ha efectuado el estudio, los alumnos son en su mayor parte de lengua catalana lo que facilita el aprendizaje de esta lengua por parte de los de lengua castellana. En una escuela donde la totalidad o la mayoría de los alumnos sean de lengua castellana aun cuando una buena parte de la enseñanza se haga en catalán, esta facilitación a través de la comunicación espontánea no se produciría. Y se puede suponer también que las familias de lengua castellana que mandan a sus hijos a escuelas en lengua catalana tienen, al menos en conjunto, una actitud favorable a esta lengua, lo que influirá en los aprendizajes lingüísticos de sus hijos, lo que tampoco puede asegurarse por principio de la totalidad de la población.

El tema de la actitud de las familias enlaza con una consideración más general. En un sistema político democrático, las decisiones sobre política linguística han de tener en cuenta las opiniones de la población representada por los partidos políticos y expresada en las elecciones. A la hora de marcar el ritmo de la expansión del catalán en la enseñanza estas opiniones han de ser tenidas en cuenta. Es cierto de todos modos que el ritmo actual de expansión es perfectamente moderada y que hasta ahora no ha tropezado con resistencias importantes.

Los resultados del estudio que he expuesto admiten una interpretación de otro orden que consiste en decir que estos resultados demuestran que la escuela puede influir pero no puede cambiar substancialmente la situación lingüística existente e incluso que en gran parte se limita a repetirla. Sólo si cambia la situación del catalán en el conjunto de la vida social de Cataluña la escuela podrá conseguir resultados más brillantes en este campo.

Argumentos de este tipo alimentan las discusiones actuales sobre la escuela en $\mathrm{Ca}$ taluña. Como este artículo no tiene directamente por objeto la política educativa no voy a ocuparme de ellos. Lo que sí en cambio intentaré hacer es mostrar que los.datos del estudio permiten otro tipo de análisis, y que los resultados de este análisis pueden ser también interesantes a la hora de establecer una política educativa.

\section{BILINGUISMO Y ACTIVIDAD INTELECTUAL}

Tanto el estudio que reseñamos como la experiencia de diversos educadores demuestran que los niños que están precozmente en contacto con una segunda lengua - niños de lengua materna catalanä que al llegar a la escuela están ya familiarizados con el catalán niños de lengua materna castellana que asisten a un.centro de preescolar catalán- adquieren esta segunda lengua aparentemente sin esfuerzo y que esta adquisición no interfiere con su desarrollo intelectual o con sus aprendizajes escolares.

La primera manifestación de este hecho lo constituye el aprendizaje de la lectura. Una vez que el niño familiarizado con las dos lenguas ha aprendido a leer en una lengua, traspasa su competencia lectora a la otra prácticamente sin esfuerzo. E incluso, no parece que influya en forma apreciable el hecho de que aprendan a leer primero en su lengua propia o primero en su segunda lengua. La razón es naturalmente que la operación de leer tiene un componente intelectual común a los aprendizajes en las dos lenguas. A lo que debe añadirse que las reglas arbitrarias que ligan las letras a los sonidos son relativamente parecidas en las dos lenguas.

Por otra parte el estudio parece indicar, aunque este hecho requeriría demostraciones más precisas, que a estos niños que adquieren pronto la segunda lengua el que reciban la enseñanza en su primera lengua o en su segunda lengua, no afecta a sus resul- 
tados académicos. Es decir, que los niños catalano-parlantes que asisten a una escuela en lengua castellana consiguen unas calificaciones académicas similares a las de sus compañeros de lengua castellana. $Y$ de modo parecido los niños castellano-parlantes que asisten a escuelas en las que la enseñanza es predominantemente en catalán consiguen calificaciones parecidas a las de sus compañeros de lengua catalana. Lo cual desmiente, como a veces se ha pensado, que la enseñanza en una segunda lengua afecta necesariamente en forma desfavorable al desarrollo intelectual y al rendimiento académico de los escolares.

Por tanto, cuando los catalano-parlantes reclamaban y reclaman que sus hijos deben recibir la enseñanza en primer lugar en catalán pueden apoyarse en muchos argumentos, pero no en el de que al no hacerlo así se atenta gravemente a su desarrollo intelectual o académico. Y del mismo modo los castellano-parlantes pueden enviar tranquilamente a sus hijos a escuelas catalanas sin sentir reparos por este motivo.

\section{CONDICIONAMIENTOS SOCIO-CULTURALES}

Lo que acabo de deducir de los resultados conseguidos en el estudio que estoy comentando coincide con los resultados de estudios bien conocidos y concretamente con los experimentos con el llamado método de inmersión popularizado por la obra de Lambert. Como es sabido cierto número de familias de lengua inglesa habitantes del Canadá francés decidieron que sus hijos debían llegar a dominar perfectamente el francés y para ello promovieron un centro de enseñanza en el que sus hijos recibían la enseñanza totalmente en francés desde el primer día. Los resultados de esta inmersión temprana fueron plenamente positivos: los niños adquirieron el francés y se convirtieron en bilingües sin que su inglés sufriese ningún daño ni dejase de progresar, y sus resultados académicos fueron comparables a los que habrían conseguido en una escuela en lengua inglesa. Tal fue la base de la popularidad del método de inmersión.

Con el tiempo el propio Lambert, su más ferviente defensor, advirtió que el método no era recomendable en todos los casos. Así por ejemplo, si a las familias de lengua francesa que viven en el Canadá francés, en nombre de las ventajas de la inmersión se les obliga a que envíen a sus hijos a escuelas exclusivamente en lengua inglesa lógicamente protestarán y con razón. Dado el peso del inglés en la vida pública, los niños en lengua inglesa que viven en el Canadá francés no corren el peligro de perder su lengua aunque asistan a una escuela francesa. Pero la recíproca no es cierta.

Lambert dedujo de aquí que la inmersión como método sólo es recomendable cuando el niño mantiene un uso familiar y público de su primera lengua que asegura su perduración. Es una recomendación perfectamente razonable. En una situación en la que coexisten una lengua fuerte y una lengua débil no comporta peligro para su primera lengua mientras que la inmersión del que habla la lengua débil en la lengua fuerte pone claramente en peligro la perduración de su primera lengua.

Pero la verdad es que los peligros de la inmersión y en general de la adquisición temprana de una segunda lengua no se limitan al peligro de pérdida de la primera lengua, a consecuencia del desequilibrio entre las dos. Incluso para el mismo par de lenguas en presencia, la inmersión y en general la educación bilingüe puede tener efectos muy diversos, en unos casos positivos y en otros negativos, según las circunstancias socio-culturales. La cuestión merece un examen atento.

Pensemos en la población de la lengua hispana en los Estados Unidos, chicanos, puertorriqueños, dominicanos, y de otras procedencias que en su mayoría ocupan estratos muy bajos de la sociedad. La educación escolar y en primer lugar la adquisición escolar del inglés debería ser el camino principal de su integración en la sociedad norteamericana. Pero para estos alumnos la escuela no cumple esta función y su bajo nivel de conocimiento del inglés constituye el primer escollo para esta integración. Ante este hecho, y para evitar este fracaso, se difundieron los programas de educación bilingüe que debían permitir una adquisición gradual de la segunda lengua y con ello un verdadero bilingüismo y con ello una integración cómoda en la nueva sociedad. 
Los defensores de la enseñanza bilingüe creyeron firmemente que, aparte del derecho de los hispanos a conservar su propia lengua, sería fácil demostrar que los alumnos que seguían programas exclusivamente en inglés obtendrán peores resultados.

Sin embargo los numerosos estudios realizados no resultan demasiado concluyentes en este sentido, no está claro que los alumnos en los programas bilingües consigan resultados académicos mucho mejores, pero sobre todo parece que se produce un claro efecto de balanza: los alumnos que más inglés adquieren son los que están menos expuestos al castellano y los que están más familiarizados con el castellano, porque están más en contacto con él, son los que menos progresan en inglés.

Hay sin embargo una excepción altamente significativa. En la mayoría de estudios realizados en Florida, donde los hispánicos son sobre todo familias de clase media inmigrados de Cuba, este efecto de balancín no se produce: los alumnos con mejores calificaciones en inglés son también los alumnos con mejores calificaciones en castellano.

Dado que las lenguas en presencia son las mismas, las diferencias en los resultados que acabamos de notar han de tener una raíz socio-cultural que actúa en contra de los más desfavorecidos.

Una primera explicación propuesta por Cummins es de tipo lingüístico. La adquisición de una segunda lengua requiere que la primera esté ya básicamente establecida y si la segunda se introduce cuando la primera es todavía débil e insegura su introducción tendrá efectos disruptivos. Dado que los niños de un nivel cultural pobre tienen un desarrollo lingüístico también pobre, la introducción de una segunda lengua al llegar a la escuela tendrá con facilidad efectos desfavorables.

Expuesta como una explicación puramente lingüística esta argumentación no resulta muy convincente. Los niños que crecen en un ambiente familiar en el que se hablan dos lenguas entran en contacto con una segunda lengua muy pronto y por supuesto antes de que la primera esté consolidada sin que esto tenga consecuencias negativas. El dato fundamental, por tanto, debe buscarse en las características peculiares del desarrollo lingüístico de los niños según el medio sociocultural en el que crecen.
La dependencia entre desarrollo lingüístico y clase social se da también en los niños monolingües, tal como puso de relieve Bernstein. Coincidamos o no con él en cuanto a la naturaleza y a la raíz de estas diferencias, creo que es fácil estar de acuerdo en que el niño de clase media llega a la escuela con una modalidad de lengua y con un uso de la lengua parecido al que rige en la escuela, mientras el niño de clases sociales más bajas llega a la escuela con una modalidad de lengua y con una forma de usarla sensiblemente distinta, y que esta diferencia constituye un hándicap inicial que difí. cilmente supera o que solo muy imperfectamente supera.

En la medida en que esto sea cierto, y por mi parte estoy convencido de que lo es, la dificultad principal del niño chicano o puertorriqueño en los EE.UU. no es tanto que la escuela hable en inglés como el que hable en un inglés escolar una modalidad de lengua y una forma de usarla para las que no puede apoyarse en sus propios usos lingüísticos, una dificultad que encontraría igualmente si la escuela hablase en castellano pero que evidentemente aumenta por el hecho de hablar en inglés. Así se explica además el efecto de balancín, cuanto más mantiene el uso de su propia lengua menos avanzará en el inglés, como es evidente, pero al mismo tiempo los progresos que logre hacer en el inglés escolar no podrán refluir sobre su castellano coloquial haciéndolo progresar. Para los hijos de los cubanos de Florida, en cambio, que hablan en su casa un castellano parecido al inglés de la escuela - un padre de clase media habla a su hijo en forma parecida a como habla el maestro a sus alumnos - no sólo el aprender el inglés escolar le resulta más fácil sino que cualquier progreso en una de las dos lenguas puede repercutir en el progreso de la otra.

Todo lo cual podría resumirse diciendo que los niños para los que la enseñanza bilingüe o la enseñanza en una segunda lengua resulta negativa o perjudicial son precisamente los niños que en una enseñanza monolingüe y en su propia lengua encontrarían también dificultades porque arrastran un hándicap lingüístico.

Las diferencias de modalidades y formas de uso de la lengua se dan entre distintas 
clases sociales y se dan también entre distintas culturas. $Y$ estas diferencias se hacen máximas cuando entre los dos grupos lingüísticos en presencia se dan a la vez diferencias sociales y culturales fuertes. Más todavía si uno de los grupos lingüísticos está sólidamente integrado y el otro más o menos culturalmente desintegrado.

En los países del Magreb actualmente coinciden dos culturas; la árabe musulmana y la occidental y, más especialmente, francófona. Y la educación es en buena parte bilingüe en árabe y en francés. Diversos investigadores y entre ellos recientemente Fitouri han hecho notar que el bilingüismo educativo tiene efectos claramente distintos según los niveles sociales. Para los hijos de las familias de un cierto nivel sociocultural, tanto si son sólidamente tradicionalistas musulmanes como si están abiertas a la cultura francesa, la introducción del francés significa un factor positivo en su progreso académico y por tanto un enriquecimiento, en cambio para los emigrantes del campo y pobladores de los suburbios ciudadanos prácticamente desgajados de su pasado tradicional, la introducción del francés representa el escollo definitivo en su camino de fracasados escolares, fracaso que no es sino el reflejo de su situación cultural, desgajados de su cultural tradicional, pero incapacitados para integrarse en las formas culturales llegadas de fuera.

Por supuesto no pretendo decir que la situación de los castellano-parlantes en el cinturón industrial de Barcelona o de los catalano-parlantes en las comarcas rurales y aisladas sea comparable a la de los chicanos o de los mogrebíes: cada situación de lenguas en contacto es distinta y tiene su propia originalidad. Pero lo que sí pretendo decir y con el mayor énfasis posible, es que todo sistema educativo que ha de tener en cuenta dos lenguas, tiene unos condicionamientos socioculturales muy fuertes y que una misma política lingüística en la educación puede conseguir resultados muy diversos se- gún las situaciones en los que se aplica. Y para comprender y aclarar estas influencias hacen falta estudios cuidadosos.

Sobre este punto la información que ofrece el estudio al que me estoy refiriendo es escasa y no podría ser de otro modo pues no era este el objetivo que se proponía. Pero creo que ha llegado la hora de proponérselo y de plantear investigaciones que intenten poner en claro la influencia que la lengua de la enseñanza tiene sobre el desarrollo intelectual y sobre los resultados académicos de los alumnos en función de la situación socio-cultural de éstos. La información que así se consiguiese sería importante para la planificación educativa en Cataluña, pero encontraría además una audiencia mucho más amplia, en un momento en que la presencia de distintas lenguas en la educación ha llegado a ser un problema candente en todo el mundo y en el que las investigaciones serias sobre el tema son escasas y están concentradas además en unos pocos lugares. Un trabajo sistemático realizado aquí se convertiría con facilidad en un punto de referencia obligado.

No quiero terminar este comentario sin hacer una observación que me parece ineludible. En Cataluña, y como consecuencia de un cambio político, se ha pasado de una situación en la que el castellano era el único vehículo de la enseñanza a una situación en la que las dos lenguas comparten con distintas fórmulas la enseñanza. Este cambio ha ocurrido en forma relativamente rápida y con una intensidad y una extensión que en este momento no tienen paralelo en Europa. A pesar de lo cual el cambio ha ocurrido, no diré que sin diferencias de opinión y sin problemas, que evidentemente los hay, pero sin que las diferencias de opinión cristalicen en tensiones y sin que los problemas se conviertan en conflictos colectivos. La prueba de madurez que así se ha dado y se está dando constituye en mi modesta opinión un excelente augurio para el futuro del proceso. 
SIGUan, M. (coord.): Lenguas y educación en el ámbito del Estado Español. Publicaciones de la Universidad de Barcelona, 1983. Generalitat de Catalunya: Cuatre anys de català a l'escola. Barcelona, 1983.

Siguan, M. (coord.): Adquisición precoz de una segunda lengua. Publicaciones de la Universidad de Barcelona, 1985.

\section{Resumen}

El artículo describe el sistema educativo en Cataluña en relación con las dos lenguas utilizadas en la enseñanza: catalán y castellano y comenta los resultados alcanzados en una investigación realizada para aclarar la influencia de esta enseñanza bilingïe sobre las competencias lingüisticas de los escolares en una y otra lengua. La principal consecuencia que se desprende de la investigación es que a pesar del gran espacio concedido al catalán la mayor presencia social del castellano continúa favoreciendo a esta lengua de tal modo que en igualdad de condiciones los niños de lengua catalana adquieren más dominio del castellano que los niños de lengua castellana dominio del catalán. Aunque la investigación se refiere sólo a la competencia lingǘstica y no al rendimiento escolar, el autor señala la conveniencia de investigar también este aspecto poniéndolo en relación con el nivel sociocultural de los alumnos.

\section{Résumé}

L'article décrit le systeme éducatif de la Catalogne et la place dans ce systeme des deux langues utilisés dans l'enseignement: catalan et castillan et examine les resultats d'une recherche récente sur l'influence de cet enseignement bilingue sur les compétences linguistiques des écoliers. Le resultat plus clair est que malgré la large place faite au catalan dans l'enseignement la plus grande presence du castillan dans la vie sociale continue à favoriser cette langue de telle façon que à circonstances egales les enfants de langue maternelle catalane acquièrent une competence plus grande en langue castillane que les enfants de langue castillane en catalan. Malgré que la recherche discuté dans l'article fait seulement référence aux compétences linguistiques et non à l'influence sur le developpement intelectuel et les resultats scolaires l'auteur signale l'importance d'étudier l'enseigne. ment bilingue sous ces aspects et de les mettre en rapport avec le niveau socioculturel des enfants. 